\title{
Partidismo: ¿el verdadero aliado de las fake news? Un análisis comparativo del efecto sobre la creencia y la divulgación
}

\author{
Partisanship: the true ally of fake news? A comparative analysis of \\ the effect on belief and spread
}

João Pedro Baptista. Universidade da Beira Interior, Portugal

joao.pedro.baptista@ubi.pt

$[\mathrm{CV}](\mathrm{C})$

Elisete Rodrigues Correia. Instituto Superior Técnico da Universidade de Lisboa, Portugal

ecorreia@utad.pt

$[\underline{\mathrm{CV}}] \odot \mathrm{G}$

Anabela Gradim Alves. Universidade da Beira Interior, Portugal

agradim@ubi.pt

$[\underline{\mathrm{CV}}]$ (1) $\mathrm{G}$

Valeriano Piñeiro-Naval. Universidad de Salamanca, España

vale.naval@usal.es

$[\underline{\mathrm{CV}}]$ (1) $\mathrm{G}$

Este artículo forma parte del trabajo desarrollado en el marco de una beca de doctorado, financiada por la Fundação para a Ciência e Tecnologia (FCT) (Ref.: SFRH/BD/145497/2019) de Portugal. Inicio de la investigación: 01/03/2020. Término de la investigación: 31/09/2021.

Cómo citar este artículo / Referencia normalizada

Baptista, J. P., Correia, E., Gradim, A \& Piñeiro-Naval, V. (2021). Partidismo: ¿el verdadero aliado de las fake news? Un análisis comparativo del efecto sobre la creencia y la divulgación. Revista Latina de Comunicación Social, 79, 23-47. https://www.doi.org/10.4185/RLCS-2021$\underline{1509}$

\section{RESUMEN}

Introducción: Tras la recomposición del parlamento portugués con la aparición de la derecha radical, este estudio explora la influencia de la orientación partidista en la creencia y difusión de fake news. Metodología: Se recurrió a una muestra exploratoria $(N=712)$, cuyos participantes fueron expuestos a 20 titulares políticamente sesgados (pro-derecha y pro-izquierda): la mitad fake news y la otra mitad noticias verdaderas. Los participantes evaluaron su credibilidad y voluntad de compartirlas en las redes sociales. Resultados: Es más probable que los partidarios de derecha crean y compartan fake news compatibles. Esta tendencia se verificó en todos los parámetros de medición del partidismo - (1) intención de voto, (2) simpatía partidaria y (3) autoubicación en la escala I-D_, en contraste con lo revelado con los partidistas de izquierda. Discusión y conclusiones: Solo los partidarios de derecha muestran una tendencia a creer más en fake news que favorecen su orientación. No ocurre lo mismo con los individuos de izquierdas. Sin embargo, es más probable que tanto los partidarios de derecha como de izquierda compartan contenido sesgado. Creemos que las 
personas con identidad de partido de derecha pueden estar más expuestas a la desinformación en Portugal, ya que la mayoría de los sitios de desinformación buscan esta audiencia específica.

PALABRAS CLAVE: Fake news; Noticias; Desinformación; Partidismo; Polarización política; Derecha; Izquierda.

\begin{abstract}
Introduction: After the recomposition of the Portuguese parliament with the emergence of the radical right, this study explores the influence of partisan orientation on the belief and dissemination of fake news. Methodology: An exploratory sample was used $(\mathrm{N}=712)$, whose participants were exposed to 20 politically biased headlines (pro-right and pro-left): half fake news and the other half true news. Participants evaluated their credibility and willingness to share them on social media. Results: Right-wing supporters are more likely to create and share compatible fake news. This trend was verified in all the measurement parameters of partisanship ((1) voting intention, (2) partisan sympathy and (3) self-placement on the I-D scale), in contrast to what was revealed with left-wing partisans. Discussion and conclusions: Only right-wing supporters show a tendency to believe more in fake news that favors their orientation. The same is not the case with left-wing individuals. However, both right-wing and left-wing supporters are more likely to share biased content. We believe that people with a right-wing party identity may be more exposed to disinformation in Portugal, as most disinformation sites seek this specific audience.
\end{abstract}

KEYWORDS: Fake news; News; Disinformation; Partisanship; Political polarization; Right-wing; Left-wing.

\title{
CONTENIDO
}

1. Introducción. 2. Partidismo y creencia en fake news. 3. La reorganización del sistema de partidos portugués. 4. Metodología. 4.1. Procedimientos. 4.2. Partidismo. 4.3. Análisis estadístico. 5. Resultados. 5.1. Relación entre la intención de voto (I-D), la creencia y la voluntad de compartir noticias y fake news. 5.2. Relación entre la intención de voto (por partido) y la creencia y la voluntad de compartir noticias y noticias falsas. 5.3. Asociación entre autoubicación en la escala política de izquierda-derecha, simpatía partidista, consumo y difusión de fake news. 6. Discusión y Conclusiones. 7. Referencias Bibliográficas

\section{Introducción}

Si bien siempre ha existido la creación y difusión de fake news, especialmente para lograr objetivos políticos e ideológicos (Burkhardt, 2017), fue con las elecciones presidenciales estadounidenses de 2016 cuando el fenómeno comenzó a ganar cobertura mediática (Wendling, 2018), habiéndose convertido en una amenaza global. Por un lado, la amplia difusión de fake news amenaza al periodismo, ya que desacredita la profesión al transformarse en un competidor más en la red y reducir la autoridad de la práctica periodística como constructora de conocimiento (Russell, 2019; Waisbord, 2018). Por otro lado, las noticias falsas han contribuido al deterioro de la democracia, normalizando la mentira en el discurso político y sirviendo como un instrumento de batalla política que ha contribuido a la ruptura de la confianza pública en las instituciones (Fernández, 2019).

Las fake news contemporáneas son un tipo de desinformación en línea que imita el formato de las noticias o informaciones tradicionales, creado intencionalmente para engañar o manipular al lector con declaraciones engañosas y/o totalmente falsas (Baptista y Gradim, 2020a). La literatura reciente apunta al caos actual de la comunicación pública (desconfianza en las instituciones, polarización política, redes sociales, desconfianza en los medios) como una justificación para el surgimiento de la era de la posverdad (Bennett y Livingston, 2018; Lewandowsky et al. 2017). 
En 2016, se estima que el $75 \%$ de los estadounidenses pueden haber creído historias falsas (Silverman y Singer-Vine, 2016). Silverman et al. (2016) revelaron una serie de páginas y sitios políticos ultrapartidistas en Facebook, a través de los cuales se difundió información falsa, engañosa y sesgada a miles de seguidores, replicada sucesivamente a través de las redes sociales. Posteriormente, en 2020, durante las elecciones estadounidenses, Donald Trump, aún como presidente de los Estados Unidos, difundió información falsa y engañosa durante el recuento de votos (Conger, 2020; Dean, 2020; Dilanian, 2020) al aseverar que este fue fraudulento, lo que contribuyó a que sus seguidores aceptaran una creencia falsa (Pennycook y Rand, 2021).

Estas sucesivas manipulaciones de la verdad, en diversos contextos, están acelerando el declive de la confianza pública en las distintas instituciones democráticas; a saber, en el periodismo, siendo la integridad de los periodistas blanco de agresiones físicas e infamias en varios países (Bennett y Livingston, 2018; MMF, 2021). Aun así, en 2020, los portugueses, junto con los finlandeses, fueron los que más confiaron en las noticias (56\%), valor superior a la media internacional (37\%) (Cardoso et al. 2020). Asimismo, 7 de cada 10 portugueses reconocen que el periodismo juega un papel importante para la sociedad, un dato que puede estar relacionado con la neutralidad políticoideológica y partidista de los medios portugueses a lo largo de los años, que ha permitido que los medios tradicionales se asocien a la profesionalidad, colocándolos fuera del ámbito del control político (Da Silva et al. 2017; Fishman, 2011; Hallin y Mancini, 2017). Sin embargo, Portugal cumple con la tendencia de creciente desconfianza en las noticias que se ha observado a nivel mundial, con la confianza cayendo del 65,6\% (2016) al 56,5\% en 2020 (Cardoso et al., 2020). Por otro lado, los portugueses se encuentran entre los más preocupados por la desinformación online (76\%), solo superados por los brasileños (88\%) en este ranking. La media internacional se sitúa en el $56 \%$, y los holandeses, eslovacos y alemanes son los menos preocupados (Cardoso et al. 2020). En Portugal, de manera similar a lo que viene ocurriendo en otros países, los sitios de desinformación están creciendo y ganando más seguidores, especialmente en Facebook (Cardoso et al. 2019; ISCTE, 2019; Pena, 2018a, 2018b, 2019b), mientras continúan con la difusión de falsedades durante las elecciones, especialmente contra el gobierno de izquierda portugués y producidas por la extrema derecha (Baptista y Gradim, 2020b).

La literatura ha aportado una serie de explicaciones, de carácter psicológico, como motivos de la difusión y consumo de este tipo de contenidos: por las fuertes emociones y sentimientos que provocan (Albright, 2017; Berger y Milkman, 2012; Bright, 2016), y también porque explotan prejuicios o simpatías sociales, políticos y públicos (Faragó et al., 2020; Van Bavel y Pereira, 2018). Los seres humanos tienden a aceptar más fácilmente argumentos que confirman sus creencias ideológicas y partidistas (Deppe et al. 2015; Gorman y Gorman, 2016), permitiendo que sus comportamientos y actitudes se vean claramente influenciados por su afiliación política. Además, la identidad partidista también influye en el comportamiento como, por caso, en la argumentación de los usuarios en las redes sociales (Ordaz et al., 2016). Además, la concordancia ideológico-partidista los motiva a buscar contenido "informativo" compatible con su visión del mundo (opinión, valores o creencias), aunque sea falso (Lee et al., 2019). Esta "homofilia" inherente a la mente humana, replicada en el universo digital, juega un papel crucial en la definición del comportamiento algorítmico de las redes sociales, creando cámaras de eco y/o burbujas de filtro (Flaxman et al., 2016; Lorenz-Spreen et al., 2020; Pariser, 2011) que no solo limitan al usuario a opiniones homogéneas, sino que también pueden promover la difusión de desinformación y la polarización política de los individuos.

Habiendo identificado el problema social y político que representan las fake news y la desinformación en general, el objetivo principal del presente trabajo es comprender la relación entre 
el partidismo y la creencia y difusión de fake news y noticias en Portugal. Creemos que esta investigación, aplicada al electorado portugués, es un aporte original y relevante a la literatura actual, claramente centrada en el escenario americano. Además, las noticias falsas en Portugal aún requieren de una mayor investigación. Con el creciente incremento de los populismos políticos (Fernández García y Salgado, 2020; Santana-Pereira y Cancela, 2021), así como el aumento de la cantidad de sitios de desinformación, amenazas a periodistas y un discurso racista y violento en el país (Alberti, 2020), consideramos que es el momento crucial para intentar comprender la relación que existe entre el consumo de noticias falsas y la identidad partidista. En suma, se espera que este estudio pueda señalar cuál de los dos grupos (con identidades de partido de izquierda o derecha) puede ser más tendente a creer y difundir fake news, y cómo el sesgo de partido influye en la forma en que juzgan las noticias y el contenido falso.

\section{Partidismo y creencia en fake news}

La forma en que procesamos la información ha sido un tema ampliamente abordado en el campo de la psicología, especialmente en lo que respecta a la capacidad cognitiva (Deppe et al., 2015; Pennycook y Rand, 2019; Roets, 2017). Los individuos con menores niveles de capacidad cognitiva, es decir, con interpretaciones más intuitivas, menos escépticas y asociadas a un esfuerzo menor, se correlacionan positivamente con una mayor tendencia o vulnerabilidad a creer en información dudosa y falsa, como es el caso de las fake news (Deppe et al., 2015; Pennycook y Rand, 2019; Roets, 2017). Por ejemplo, en el escenario estadounidense, los conservadores son menos calculadores que los liberales (Deppe et al., 2015; Jost et al., 2003; Swire et al., 2017) y es más probable que crean y compartan fake news (Pennycook y Rand, 2019). Por otro lado, el razonamiento motivado (como la identidad partidista) también ha sido uno de los factores más influyentes en la valoración de la información (Van Bavel y Pereira, 2018) y la formación de la opinión pública (Bolsen et al., 2014; Leeper y Slothuus, 2014), cambiando el juicio y el comportamiento político de las personas. Esta motivación ideológico-partidista, también relacionada con los aspectos cognitivos, promueve la protección de opiniones, creencias y valores preexistentes, motivando una interpretación sesgada de los asuntos políticos, incluso cuando se corrige la información falsa (verificación de hechos) (Shin y Thorson, 2017).

En este sentido, Faragó et al. (2020) mostraron que los partidarios de las políticas gubernamentales en Hungría eran más susceptibles a la creencia en fake news frente a los más críticos con el gobierno, y viceversa. En la escena política estadounidense, una vez más, Uscinski et al. (2016) argumentan que los republicanos tienden a rechazar la teoría de que el presidente Bush fue cómplice de los ataques terroristas del 11 de septiembre, mientras que es más probable que los demócratas la crean. Lo mismo ocurre, a la inversa, con la falsa teoría de que el presidente Obama no nació en Estados Unidos. Otros estudios también han apoyado esta perspectiva, al reparar en que la identidad de partido puede influir tanto en las personas de izquierda como en las de derecha (Allcott y Gentzkow, 2017; Nyhan y Reifler, 2010; Pereira y Van Bavel, 2018; Uscinski et al., 2016). Con respecto a las noticias falsas no políticas, por caso, Pereira y Van Bavel (2018) sostienen que los republicanos son más propensos a compartir fake news en comparación con los demócratas.

En general, los individuos de derecha, especialmente de extrema derecha parecen más vulnerables a las fake news tanto en creencias como en difusión (Baptista y Gradim, 2020a; Halpern et al., 2019; Lewis y Marwick, 2017; Marwick, 2018; Recuero y Gruzd, 2019). Por un lado, la desinformación online se vincula con una visión conservadora y extremista, asociada a medidas radicales contra la corrupción (Baptista y Gradim, 2020b; ISCTE, 2019), las minorías sociales, sexuales y raciales o la inmigración y el Islam (Câncio, 2020; Humprecht, 2019). Por otro lado, la narrativa populista de derecha se asemeja a la narrativa persuasiva que se repite en las fake news (Baptista, 2020). Cabe 
señalar que la derecha radical etiquetó como "noticias falsas" aquellas que no favorecían a su líder político ni a sus ideologías. La legitimidad periodística fue entonces cuestionada no solo por el discurso de Trump en los Estados Unidos, sino también por la derecha alemana radical (por ejemplo, por el movimiento anti-refugiados PEGIDA: Patriotas Europeos contra la Islamización de Occidente y el partido Alternativa para Alemania) (Brauck et al., 2016). En Portugal, la derecha radical portuguesa también ha amenazado a periodistas [1], un hecho que puede estar asociado a la difusión y consumo de desinformación online (Pena 2018b, 2018a, 2019; Câncio 2020; Baptista y Gradim, 2020b), la actividad de las milicias digitales y la actuación masiva de perfiles falsos que buscan imponer la ideología del partido Chega (Carvalho, 2020b; Silva, 2020).

\section{La reorganización del sistema de partidos portugués}

Hasta las anteriores elecciones legislativas portuguesas (2019), la mayoría de los partidos que tenían representación parlamentaria (los más relevantes para el sistema de partidos, Sartori, 2005), existía desde la instauración de la democracia, hace más de 40 años. Esto demuestra que hubo una rápida consolidación del sistema de partidos, ya que su período de formación se refiere a los años inmediatamente posteriores a la Revolución de 1974. La dicotomía izquierda y derecha ha servido para enmarcar un sistema político multipartidista, con alternancia de gobierno entre dos partidos moderados: Partido Socialista (PS), de centroizquierda, y Partido Social Demócrata (PSD), de centroderecha, salvo algunas coaliciones entre el PSD y el Centro Democrático Social-Partido Popular (CDS-PP) del ala más a la derecha (Jalali y Cabral, 2003; Lisi, 2016, 2019). El sistema político se constituía, pues, por la izquierda radical tradicional (y más conservadora) del Partido Comunista Portugués (PCP) (Freire, 2017; March, 2012; Pereira, 2016), la nueva izquierda del "Bloco de Esquerda" (BE) (Lisi, 2013) y, más recientemente, "Pessoas-Animais-Natureza" (PAN) vinculados a la causa animal y ambiental (Catarino, 2017); en el centroizquierda el PS, el centroderecha por el PSD (Guedes, 2012, 2016) y a la derecha, en el ala más conservadora, el CDSPP (Jalali y Cabral, 2003; Lisi, 2016, 2019).

En 2019, tres nuevos partidos de reciente creación se unieron al sistema político portugués. El Partido Livre (L) a la izquierda, la Iniciativa Liberal (IL) y Chega $(\mathrm{CH})$ a la derecha. Por tanto, Livre parece ocupar una izquierda libertaria, europeísta y en la línea ideológica del Green New Deal (Fernandes y Magalhaes, 2020); IL se erige en partido de derecha liberal en términos económicos y sociales (Fernandes y Magalhaes, 2020; Lisi, 2019), pero sobre todo con un enfoque especial en la desregulación del mercado, la privatización y la reducción del papel del Estado en la agenda económica (Jalali at ál., 2021). Por último, el surgimiento del CH termina con la excepción, que se ha observado hasta entonces en Portugal, del fracaso de los partidos políticos populistas y antisistema (Carreira da Silva y Salgado, 2018), haciendo que la derecha radical logre una visibilidad nunca vista hasta entonces (Costa, 2011; de Almeida, 2015). La representación parlamentaria de Chega, incluso con un solo diputado, representó el surgimiento de la derecha populista radical en Portugal (Lisi et al., 2021; Mendes y Dennison, 2020), que puede tener que ver con el pobre desempeño electoral no solo de los partidos centristas, sino también de los partidos de derecha (Lisi et al., 2021). Chega se asemeja a otros partidos de la derecha radical, como Vox, en España, especialmente en el tratamiento de asuntos como la inmigración y el islam (Mendes y Dennison, 2020). Si hasta las elecciones legislativas anteriores, PS y PSD recibieron la etiqueta de "partidos catch-all", por la forma en que se han mantenido en el poder y buscan maximizar los votos (Belchior, 2015; Jalali y Cabral, 2003), CH tiene el objetivo de "capturar el mayor número de personas descontentas en todo el espectro político y la abstención" (Marchi, 2019), con una narrativa antisistema que movilice a los votantes de todos los partidos (Lisi et al., 2021) y un programa que defienda sentencias más duras para delitos más graves y una mayor seguridad (Fernandes y Magalhaes, 2020; Mendes y Dennison, 2020). 
De esta forma, el sistema de partidos portugués sufrió una reformulación de todo el espectro político, pero con especial énfasis en la derecha. Aun así, la representación de partidos pequeños no parece afectar, por el momento, a la hegemonía de los partidos del arco de gobernanza (Lisi et al., 2021).

\section{Metodología}

Para lograr los objetivos propuestos al inicio del trabajo, se recurrió a la aplicación de un cuestionario que buscaba conocer la capacidad del electorado portugués para distinguir las fake news de las noticias, expresando su opinión sobre el nivel de credibilidad de los titulares presentados y su voluntad de compartir estas informaciones con sus amigos. Se envió un cuestionario autoadministrado y en línea a portugueses mayores de 18 años. La muestra final se compone de 712 participantes de todas las regiones del país y estuvo disponible durante seis meses (entre marzo y septiembre de 2020). Este estudio continúa la investigación iniciada por Baptista et al. (2021). Por tanto, analizamos a los mismos participantes $(\mathrm{N}=712)$. Sin embargo, el presente análisis se centra en una parte diferente del cuestionario en relación con la identidad de los encuestados y la orientación partidista. En el estudio anterior (Baptista et al., 2021), los participantes fueron clasificados ideológicamente de acuerdo con sus actitudes y valores morales, religiosos, sociales y socioeconómicos. En la presente investigación buscamos entender cómo el partidismo - (1) intención de voto, (2) simpatía por el partido y (3) autoubicación en la escala política izquierdaderecha- se relaciona con la creencia y difusión de fake news. Los encuestados se distribuyen, por lo tanto, del siguiente modo: 34,4\% hombres y 65,6\% mujeres, con 23,6\% con escolaridad básica, $37,5 \%$ con grado universitario, $25,9 \%$ tienen máster y $13 \%$ doctorados. En cuanto a la edad de los encuestados, el 40,6\% tiene entre 18 y 30 años; $23 \%$ entre 31 y 40 años, y 36,4\% tiene más de 41 años.

\subsection{Procedimientos}

Para comprender la propensión de los portugueses al consumo de fake news, se invitó a los participantes a indicar el nivel de credibilidad y disposición para compartir titulares de fake news y noticias en un formato de publicación de Facebook (foto, título, firma y fuente, como en la Imagen 1 y en la Imagen 2) (Baptista et al., 2021). Este método ya se ha utilizado con éxito en otros estudios (Bronstein et al., 2019; Pennycook y Rand, 2019).

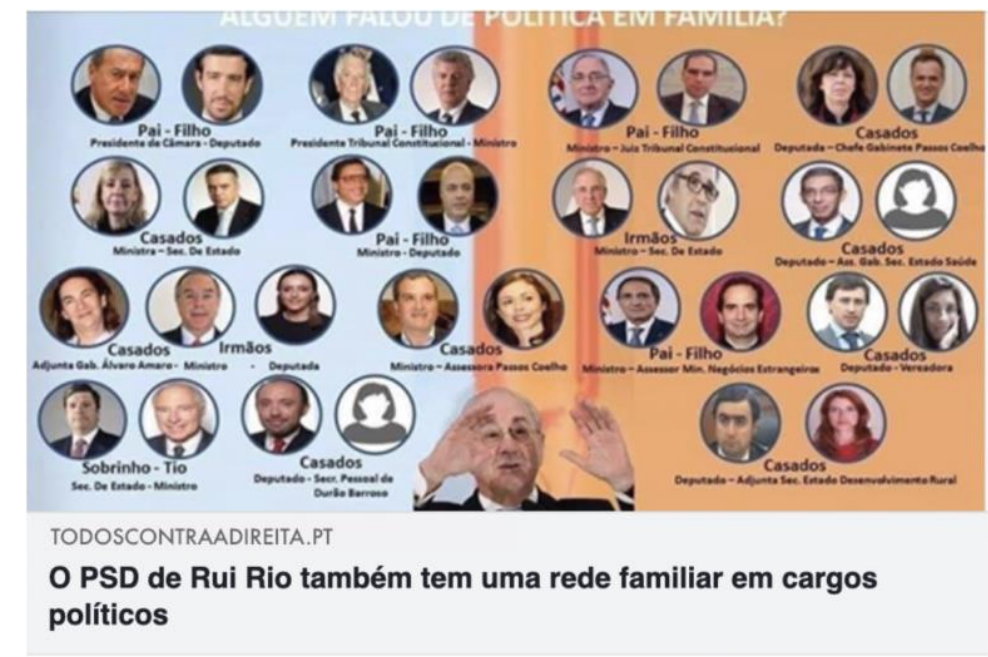

Imagen 1: Ejemplo de una noticia falsa pro-izquierda en formato de publicación de Facebook 
Fuente: Adaptado del sitio web de verificación de hechos (Polígrafo). Disponible en: https://bit.ly/3jvJC8N

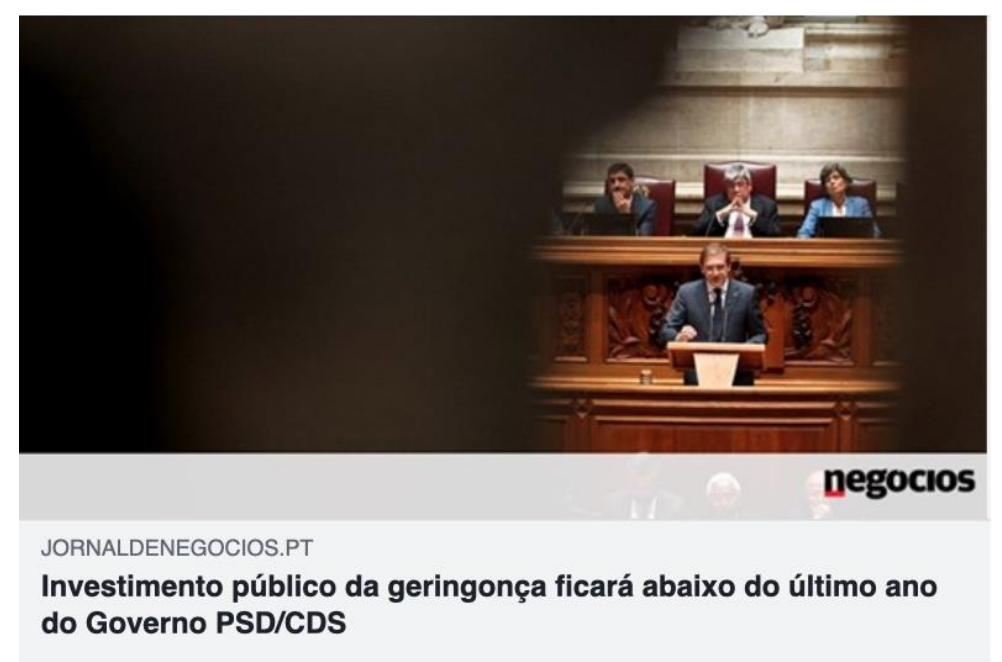

Imagen 2: Ejemplo de una noticia pro-derecha en formato de publicación de Facebook Fuente: Imagen adaptada de jornaldenegocios.pt. Disponible en: https://bit.ly/2OiaxcO

El cuestionario se dividió en 2 secciones: (1) exposición aleatoria a titulares y fake news, (2) cuestiones relacionadas con la identidad y orientación partidista de los participantes (intención de voto, grado de simpatía por los diferentes partidos y autoubicación en la escala izquierda-derecha).

Por lo tanto, cuando eran expuestos a titulares de fake news y noticias, las personas tenían que responder a dos preguntas por cada titular: P1. "Según su conocimiento, ¿cómo califica el siguiente titular?" En una escala de 5 puntos $(1=$ nada creíble, $2=$ poco creíble, $3=$ algo creíble, $4=$ bastante creíble, 5 = muy creíble) y P2. “¿Cuál es su disposición para compartir el siguiente titular?' ( 1 = nada de voluntad, 2 = poca voluntad, 3 = algo de voluntad, $4=$ mucha voluntad). Estas preguntas se realizaron para cada uno de los 20 titulares presentados: 10 fake news (5 pro izquierda y 5 pro derecha) y 10 noticias ( 5 pro izquierda y 5 pro derecha), con el fin de establecer una relación partidista con la creencia y difusión de noticias y fake news (ver Tabla 1). Todos los titulares falsos que usamos fueron tomados de Polígrafo [2], un verificador de hechos en línea portugués. La noticia real se seleccionó de fuentes de noticias que tenían titulares inusuales, con el fin de requerir el mismo esfuerzo de los participantes cuando fueron cuestionados (Baptista et al., 2021). 
RLCS, Revista Latina de Comunicación Social, 79, 23-47

[Investigación] DOI: 10.4185/RLCS-2021-1509 | ISSN 1138-5820| Año 2021

Tabla 1. Titulares de fake news y noticias que fueron usadas en cuestionario.

\begin{tabular}{|c|c|c|}
\hline & Titular & $\begin{array}{l}\text { Adaptación/Fuente } \\
\text { (URL’s) }\end{array}$ \\
\hline \multirow{5}{*}{ 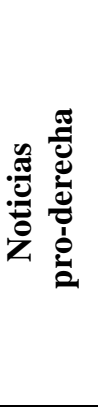 } & $\begin{array}{l}\text { El PCP obliga a sus diputados a donar parte de su } \\
\text { sueldo al partido }\end{array}$ & \\
\hline & $\begin{array}{l}\text { La inversión pública de "geringonça" estará por debajo } \\
\text { del último año del Gobierno PSD / CDS }\end{array}$ & https://bit.ly/2OiaxcO \\
\hline & $\begin{array}{l}\text { "En estos cuatro años, tuvimos la mayor carga fiscal de } \\
\text { la historia" }\end{array}$ & $\underline{\text { https://bit.ly/3cUffHU }}$ \\
\hline & $\begin{array}{l}\text { El portavoz de ANTRAM es miembro del PS y fue } \\
\text { designado para dos cargos por el actual Gobierno }\end{array}$ & https://bit.ly/3jC6tQg \\
\hline & $\begin{array}{l}\text { El nuevo ejecutivo de Costa ha tenido la mayor } \\
\text { cantidad de ministros desde } 1976\end{array}$ & https://bit.ly/36QnMaY \\
\hline \multirow{5}{*}{ そ气 } & $\begin{array}{l}\text { Diputado del PSD llama "mierda" a la clase de } \\
\text { orientación sexual y motiva la denuncia de BE }\end{array}$ & https://bit.ly/36Qo7KM \\
\hline & $\begin{array}{l}\text { Hace } 28 \text { años, que no había tan pocos desempleados } \\
\text { inscritos }\end{array}$ & https://bit.ly/3aIsfhb \\
\hline & $\begin{array}{l}\text { Bloco es el único partido parlamentario con cuentas sin } \\
\text { irregularidades }\end{array}$ & https://bit.ly/3jumgAi \\
\hline & $\begin{array}{l}\text { Hijo de Durão Barroso fue contratado por Banco de } \\
\text { Portugal "por invitación y sin licitación" }\end{array}$ & https://bit.ly/3juxVPD \\
\hline & Hace 40 años, el PSD falló la ley que creó el SNS & https://bit.ly/3jC8koc \\
\hline \multirow{5}{*}{ 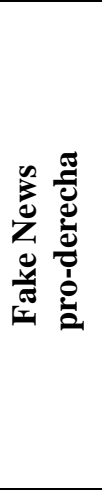 } & $\begin{array}{l}\text { Con la "geringonça" en el poder, el precio del barril de } \\
\text { petróleo bajó un } 50 \% \text { mientras que el precio del diésel } \\
\text { subió un } 40 \%\end{array}$ & https://bit.ly/3q2GNP8 \\
\hline & $\begin{array}{l}\text { Catarina Martins defiende RSI de } 750 \text { euros "para } \\
\text { gitanos, migrantes y otros" }\end{array}$ & https://bit.ly/3cP4PcB \\
\hline & $\begin{array}{l}\text { Joacine Katar Moreira do Livre solo empezó a } \\
\text { tartamudear en esta campaña electoral }\end{array}$ & https://bit.ly/3p1i9gj \\
\hline & $\begin{array}{l}\begin{array}{l}\text { Programa electoral del PAN "propone perdón a } \\
\text { violadores sexuales" }\end{array} \\
\end{array}$ & https://bit.ly/3cS9RoF \\
\hline & $\begin{array}{l}\text { El Partido Socialista no quiere que los portugueses } \\
\text { sepan quiénes son los políticos que reciben } \\
\text { subvenciones vitalicias }\end{array}$ & https://bit.ly/3cOWdTn \\
\hline \multirow{5}{*}{ 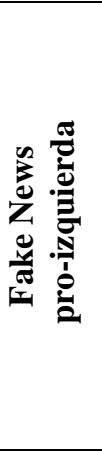 } & $\begin{array}{l}\text { Joacine Katar Moreira es la primera diputada negra en } \\
\text { Portugal }\end{array}$ & https://bit.ly/3oWMooR \\
\hline & $\begin{array}{l}\text { Estadísticas de Portugal: más de la mitad de los } \\
\text { puestos de trabajo creados desde } 2015 \text { tienen salarios } \\
\text { superiores a } 1200 €\end{array}$ & https://bit.ly/3a0jrE7 \\
\hline & $\begin{array}{l}\text { El PSD de Rui Rio también tiene una red familiar en } \\
\text { cargos políticos }\end{array}$ & https://bit.ly/3jvJC8N \\
\hline & $\begin{array}{l}\text { El PSD votó en contra de la reducción del IVA a la } \\
\text { electricidad que ahora también propone }\end{array}$ & https://bit.ly/3q5uLEK \\
\hline & $\begin{array}{l}\text { El gobierno redujo las deudas del SNS con los } \\
\text { proveedores de hospitales en un } 50 \%\end{array}$ & https://bit.ly/3d0LncK \\
\hline
\end{tabular}

Fuente: elaboración propia

\subsection{Partidismo}

La orientación partidista del electorado se midió con arreglo a tres parámetros: (1) intención de voto en las elecciones, (2) grado de simpatía por cada partido político con escaño parlamentario en la Asamblea de la República, y (3) autoubicación en la escala política izquierda-derecha. De esta forma, 
se invitó a los participantes a posicionarse en una escala de 10 puntos, donde $1=$ izquierda y $10=$ derecha.

En cuanto a la intención de voto, se planteó la siguiente pregunta: "Si mañana hubiera elecciones para la Asamblea de la República, ¿a qué partido votaría?”. Los encuestados tenían 9 posibles opciones de respuesta: de izquierda, Partido Comunista Portugués (PCP), Bloco de Esquerda (BE), Pessoas-Animais-Natureza (PAN), Livre (L), Partido Socialista (PS); de derecha, Partido Social Demócrata (PSD), Centro Democrático Social (CDS), Iniciativa Liberal (IL), Chega (CH). Considerando 566 respuestas válidas, el PS sería el partido con más votos con un 30,7\%, seguido de PSD (18,6\%), BE (17,7\%), CH (9,4\%), PAN (9,4\%), IL (4,8\%), L (3,5\%), PCP $(3,5 \%)$ y CDS $(2,5 \%)$.

En cuanto a la medición de la simpatía partidaria, los encuestados debían, en una escala de 4 puntos ( 1 = nada de simpatía, 2 = poca simpatía, 3 = algo de simpatía, 4 = mucha simpatía), expresar su relación con cada uno de los partidos marcados. El PS fue, en promedio, el partido que mayor simpatía generó en los votantes $(M=2,43 ; D T=0,93)$; mientras $\mathrm{CH}$ fue, por el contrario, el partido por el cual los votantes expresaron la menor simpatía $(M=1,51 ; D T=0,84)$.

\subsection{Análisis estadísticos}

Inicialmente se llevaron a cabo procedimientos de estadística descriptiva de tendencial central (media) y dispersión (desviación típica) respecto a las variables evaluadas. Para verificar el supuesto de normalidad de los datos, se realizó el análisis de simetría de la distribución de frecuencias (normalidad univariante) utilizando los coeficientes de asimetría (asimetría) y curtosis (aplanamiento). A través del análisis de estos valores, se encontró adecuado el uso de pruebas paramétricas. Así pues, se recurrió al análisis de varianza multivariado (MANOVA) seguido, siempre que fue posible, del análisis de varianza (ANOVA), y se utilizaron pruebas de comparaciones múltiples de los promedios para determinar qué diferencias existen entre el partidismo, la creencia y la difusión de noticias y noticias falsas. La asociación entre variables se midió mediante el coeficiente de correlación de Pearson $(r)$. Todos los análisis estadísticos se realizaron utilizando el programa SPSS v. 27.0 (IBM SPSS 27.0, Chicago, IL). En todos los análisis estadísticos se consideraron valores de significación de $p<0,05$.

\section{Resultados}

El objetivo principal de este estudio fue evaluar la influencia de la identidad de partido del electorado portugués en la creencia y difusión de fake news y noticias políticas. Nuestros resultados indican, en general, que los participantes tenían, en promedio, valores más altos de creencia en relación con las noticias en comparación con los niveles de creencia en fake news, como se muestra en la Tabla 2. 
RLCS, Revista Latina de Comunicación Social, 79, 23-47

[Investigación] DOI: 10.4185/RLCS-2021-1509 | ISSN 1138-5820 | Año 2021

Tabla 2. Medidas descriptivas y normalidad univariante

\begin{tabular}{|c|c|c|c|c|}
\hline \multirow[t]{2}{*}{ Variables } & \multicolumn{4}{|c|}{$\begin{array}{c}\text { Muestra } \\
\mathrm{N}=712\end{array}$} \\
\hline & $\mathrm{M} \pm \mathrm{DT}$ & Sk & $\mathrm{Ku}$ & Secuencia \\
\hline Aceptación de noticias $^{\mathrm{a}}$ & $2,53 \pm 0,63$ & 0,55 & 0,22 & $1-5$ \\
\hline Aceptación de noticias pro-derecha ${ }^{\mathrm{a}}$ & $1,70 \pm 0,70$ & 1,07 & 0,74 & $1-5$ \\
\hline Aceptación de noticias pro-izquierda ${ }^{a}$ & $2,07 \pm 0,75$ & 0,33 & $-0,44$ & $1-5$ \\
\hline Aceptación de fake news ${ }^{a}$ & $1,89 \pm 0,64$ & 0,42 & $-0,45$ & $1-5$ \\
\hline Aceptación de fake news pro-derecha ${ }^{\mathrm{a}}$ & $2,48 \pm 0,69$ & 0,66 & 0,24 & $1-5$ \\
\hline Aceptación de fake news pro-izquierda ${ }^{a}$ & $2,60 \pm 0,74$ & 0,58 & 0,39 & $1-5$ \\
\hline Voluntad de compartir noticias ${ }^{\mathrm{b}}$ & $1,52 \pm 0,58$ & 1,12 & 0,95 & $1-4$ \\
\hline Voluntad de compartir noticias pro-derecha ${ }^{\mathrm{b}}$ & $1,52 \pm 0,62$ & 1,32 & 1,52 & $1-4$ \\
\hline Voluntad de compartir noticias pro-izquierda ${ }^{\mathrm{b}}$ & $1,53 \pm 0,62$ & 1,16 & 0,79 & $1-4$ \\
\hline Voluntad de compartir fake news ${ }^{b}$ & $1,34 \pm 0,47$ & 1,58 & 2,44 & $1-4$ \\
\hline Voluntad de compartir fake news pro-derecha ${ }^{\mathrm{b}}$ & $1,29 \pm 0,47$ & 2,01 & 4,22 & $1-4$ \\
\hline Voluntad de compartir fake news pro-izquierda ${ }^{\mathrm{b}}$ & $1,39 \pm 0,55$ & 1,43 & 1,37 & $1-4$ \\
\hline Autoubicación en la escala izquierda-derecha & $4,80 \pm 1,94$ & 0,22 & $-0,05$ & $1-10$ \\
\hline Simpatía por los partidos politicos ${ }^{\mathrm{d}}$ & $1,93 \pm 0,49$ & $-0,08$ & 0,26 & $1-4$ \\
\hline Simpatía por los partidos politicos en derecha ${ }^{\mathrm{d}}$ & $1,66 \pm 0,63$ & 0,84 & 0,16 & $1-4$ \\
\hline Simpatía por los partidos politicos en izquierda ${ }^{\mathrm{d}}$ & $1,92 \pm 0,70$ & 0,21 & $-0,89$ & $1-4$ \\
\hline
\end{tabular}

Fuente: elaboración propia

Nota: Siglas utilizadas: $\mathrm{M}=$ Media, $\mathrm{DT}=$ Desviación típica, $\mathrm{Sk}=$ Asimetría y $\mathrm{Ku}=$ Curtosis

Sin embargo, si analizamos los valores promedio de creencias por separado, encontramos que las fake news pro-izquierda y las fake news pro-derecha tienen una mayor aceptación que las noticias a favor de la izquierda y la derecha, respectivamente. Además, la aceptación de fake news a favor de la izquierda tiene un valor de creencia más alto, incluso que la aceptación de las noticias analizadas en conjunto. Nuestros resultados también indican que la disposición a compartir es mayor en todas las noticias, en comparación con las fake news. La Tabla 2 muestra que hay, en promedio, mayor simpatía por los partidos de izquierda que por los partidos de derecha y que este es un electorado que se considera moderado, ubicándose cerca del punto 5 de la escala política.

\subsection{Relación entre la intención de voto (I-D), la creencia y la voluntad de compartir noticias y fake news}

Para evaluar si la identidad del partido, manifestada a través de su (1) intención de voto, tiene un efecto estadísticamente significativo sobre la creencia y la voluntad de compartir noticias y fake news, se realizaron análisis multivariados de varianza. En cuanto a la creencia, los resultados apuntan a la existencia de diferencias estadísticamente significativas ( $\lambda$ de Wilk $=0,814 ; p<0,001)$. El análisis univariado de varianza reveló que existen diferencias significativas solo en relación con la creencia en fake news a favor de la derecha (FND) $\left(F_{(1,564)}=20,720 ; p<0.001\right)$ y en las noticias a favor de la derecha $(\mathrm{NVD})\left(F_{(1,564)}=28,649 ; p<0,001\right)$, como podemos observar en la Tabla 3 . 
RLCS, Revista Latina de Comunicación Social, 79, 23-47

[Investigación] DOI: 10.4185/RLCS-2021-1509 | ISSN 1138-5820 | Año 2021

Tabla 3. Medias (M), desviaciones típicas (DT) y efectos univariados de la creencia y la voluntad de compartir noticias falsas (pro-izquierda y pro-derecha) por intención de voto

\begin{tabular}{lcccc}
\hline Variable & $\begin{array}{c}\text { Voto partido } \\
\text { izquierda } \\
(\mathrm{N}=367) \\
M \pm D T\end{array}$ & $\begin{array}{c}\text { Voto partido } \\
\text { derecha } \\
(\mathrm{N}=199)\end{array}$ & & \\
\hline FNE & $2,08 \pm 0,75$ & $2,02 \pm 0,73$ & 0,878 & 0,349 \\
FND & $1,55 \pm 0,60$ & $1,95 \pm 0,77$ & 45,987 & $<0,001$ \\
NVD & $2,43 \pm 0,63$ & $2,90 \pm 0,82$ & 57,089 & $<0,001$ \\
NVE & $2,49 \pm 0,70$ & $2,49 \pm 0,66$ & 0,000 & 0,998 \\
SFNE & $1,45 \pm 0,58$ & $1,33 \pm 0,48$ & 6,645 & 0,010 \\
SFND & $1,25 \pm 0,46$ & $1,40 \pm 0,52$ & 11,766 & 0,001 \\
SNVD & $1,47 \pm 0,58$ & $1,67 \pm 0,70$ & 13,805 & $<0,001$ \\
SNVE & $1,60 \pm 0,66$ & $1,48 \pm 0,56$ & 5,124 & 0,024 \\
\hline
\end{tabular}

Fuente: elaboración propia

En cuanto a la relación entre la intención de voto y el deseo de compartir noticias y fake news, los datos indican que existen diferencias significativas ( $\lambda$ de Wilk $=0,856 ; p<0,001)$, y en el caso de esta relación se dan con respecto a todas las variables: la voluntad de compartir noticias falsas proizquierda (SFNE), pro-derecha (SFND) y la voluntad de compartir noticias reales pro-izquierda (SNVE) y pro-derecha (SNVE) (Tabla 3).

En la Tabla 3 también podemos ver que los participantes que votarían a los partidos de la derecha son los que tienen los valores promedio más altos no solo en relación con la creencia en fake news pro-derecha (FND), sino también en noticias pro-derecha (NVD). Cabe señalar que los votantes de los partidos de izquierda presentan una media ligeramente superior en las fake news pro-izquierda (FNE) que los votantes de derecha; sin embargo, esta diferencia no es significativa $(p=0.349)$. Además, lo mismo ocurre con la voluntad de compartir. Si bien los votantes de izquierda tienen valores más altos en relación con la voluntad de compartir fake news pro-izquierda (SFNE) y noticias pro-izquierda (SNVE), los partidarios de los partidos de derecha han mostrado una mayor disposición a compartir contenido falso y verdadero a favor de la derecha.

\subsection{Relación entre la intención de voto (por partido) y la creencia y la voluntad de compartir noticias y noticias falsas}

Posteriormente, realizamos un análisis de varianza multivariado para determinar si la intención de voto (por partido) influye significativamente en la creencia y la voluntad de compartir noticias y fake news. Los resultados muestran diferencias estadísticamente significativas en cuanto a la creencia en noticias y fake news ( $\lambda$ de Wilk $=0,715 ; p<0,001)$. Los análisis univariados indicaron que había diferencias significativas entre la intención de voto (por partido) en la creencia en FND $(F(8,557)=5.058 ; p<$ $0,001)$ y en la creencia en NVD $\left(F_{(8,557)}=4,712 ; p<0,001\right)$ (ver Tabla 4). La disposición a compartir estas informaciones también arrojó diferencias significativas $(\lambda$ de Wilk $=0,782 ; p<0,001)$ entre todas las variables, como se muestra en la Tabla 4. 
RLCS, Revista Latina de Comunicación Social, 79, 23-47

[Investigación] DOI: 10.4185/RLCS-2021-1509 | ISSN 1138-5820 | Año 2021

Tabla 4. Medias (M), desviaciones típicas (DT) y efectos univariados de la creencia y la voluntad de compartir noticias falsas (pro-izquierda y pro-derecha) por intención de voto en cada partido

\begin{tabular}{|c|c|c|c|c|c|c|c|c|c|}
\hline & & FNE & FND & NVD & NVE & SFNE & SFND & SNVD & SNVE \\
\hline \multirow{6}{*}{ } & & $M \pm D T$ & $M \pm D T$ & $M \pm D T$ & $M \pm D T$ & $M \pm D T$ & $M \pm D T$ & $M \pm D T$ & $M \pm D T$ \\
\hline & PCP & $2,22 \pm 0,71$ & $1,53 \pm 0,69$ & $2,39 \pm 0,64$ & $2,56 \pm 0,71$ & $1,50 \pm 0,52$ & $1,29 \pm 0,55$ & $1,38 \pm 0,52$ & $1,58 \pm 0,69$ \\
\hline & $\mathrm{BE}$ & $1,99 \pm 0,71$ & $1,48 \pm 0,57$ & $2,39 \pm 0,60$ & $2,54 \pm 0,71$ & $1,41 \pm 0,56$ & $1,21 \pm 0,46$ & $1,44 \pm 0,58$ & $1,64 \pm 0,68$ \\
\hline & PAN & $2,02 \pm 0,78$ & $1,53 \pm 0,62$ & $2,48 \pm 0,52$ & $2,31 \pm 0,57$ & $1,30 \pm 0,55$ & $1,18 \pm 0,42$ & $1,39 \pm 0,49$ & $1,38 \pm 0,48$ \\
\hline & $\mathrm{L}$ & $2,13 \pm 0,66$ & $1,50 \pm 0,43$ & $2,51 \pm 0,64$ & $2,49 \pm 0,55$ & $1,44 \pm 0,58$ & $1,24 \pm 0,40$ & $1,44 \pm 0,53$ & $1,54 \pm 0,60$ \\
\hline & PS & $2,14 \pm 0,78$ & $1,60 \pm 0,62$ & $2,44 \pm 0,68$ & $2,52 \pm 0,75$ & $1,52 \pm 0,61$ & $1,30 \pm 0,46$ & $1,52 \pm 0,62$ & $1,66 \pm 0,69$ \\
\hline \multirow{6}{*}{ 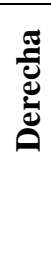 } & PSD & $1,96 \pm 0,76$ & $1,80 \pm 0,71$ & $2,83 \pm 0,82$ & $2,53 \pm 0,71$ & $1,29 \pm 0,46$ & $1,31 \pm 0,45$ & $1,63 \pm 0,69$ & $1,46 \pm 0,55$ \\
\hline & IL & $1,93 \pm 0,78$ & $1,67 \pm 0,61$ & $2,73 \pm 0,75$ & $2,29 \pm 0,53$ & $1,25 \pm 0,48$ & $1,29 \pm 0,46$ & $1,65 \pm 0,61$ & $1,34 \pm 0,38$ \\
\hline & CDS & $1,98 \pm 0,77$ & $1,71 \pm 0,49$ & $2,57 \pm 0,66$ & $2,22 \pm 0,48$ & $1,52 \pm 0,60$ & $1,48 \pm 0,48$ & $1,51 \pm 0,48$ & $1,41 \pm 0,36$ \\
\hline & $\mathrm{CH}$ & $2,22 \pm 0,62$ & $2,45 \pm 0,81$ & $3,23 \pm 0,82$ & $2,60 \pm 0,62$ & $1,39 \pm 0,50$ & $1,61 \pm 0,62$ & $1,83 \pm 0,81$ & $1,59 \pm 0,68$ \\
\hline & $F$ & 1,220 & 12,021 & 9,578 & 1,324 & 2,244 & 3,983 & 2,690 & 2,080 \\
\hline & $p$ & $p=0,285$ & $p<0,001$ & $p<0,001$ & $p=0,228$ & $p=0,023$ & $p<0,001$ & $p=0,007$ & $p=0,036$ \\
\hline
\end{tabular}

Fuente: elaboración propia

En cuanto a la creencia en NVD, las pruebas de comparación múltiple mostraron diferencias estadísticamente significativas entre los partidarios de $\mathrm{CH}$ y todos los demás, teniendo los votantes de $\mathrm{CH}$ valores promedio más altos. También hay diferencias significativas entre los partidarios de $\mathrm{BE}$ con $\mathrm{CH}(p<0,001)$, PSD $(p<0,001)$, IL $(p=0,024)$; entre los partidarios del PCP y los que votarían por $\mathrm{CH}(p<0,001)$ y PSD $(p=0,010)$; entre los partidarios del PS con CH $(p<0,001)$, PSD $(p<0,001)$, $\mathrm{IL}(p=0,044)$; Partidarios del PAN con $\mathrm{CH}(p<0,001)$ y PSD $(p=0,004)$; votantes en L y votantes en $\mathrm{CH}(p<0,001)$. Cabe señalar que todos los votantes de los partidos de derecha tienen valores más altos que los partidarios de la izquierda en cuanto a noticias que favorecen a la derecha.

Con respecto a la creencia en fake news pro-derecha (FND), se observa un comportamiento similar. Es decir, los partidarios de $\mathrm{CH}$ son significativamente diferentes de todos los demás y también tienen valores promedio de creencias más altos. También hay diferencias significativas entre los votantes de izquierda y los votantes de derecha: BE-CH $(p<0,001)$, BE-PSD $(p=0,010)$; PCP-CH $(p<0,001)$; PS-CH $(p<0,001)$, PS-PSD $(p=0,013)$; PAN-CH $(p<0,001)$, PAN-PSD $(p=0,013)$, L-CH $(p<$ $0,001)$. Por último, son los votantes de los partidos de derecha los que tienen los valores más altos.

En cuanto a la voluntad de compartir contenido y el voto en cada partido, las pruebas de comparación múltiple demuestran un comportamiento de los votantes similar al verificado en relación con la creencia. En cuanto a la disposición a compartir fake news pro-izquierda (SFNE), existen diferencias estadísticamente significativas entre votantes que votaron por el PS con votantes en PSD $(p<0,001)$, PAN $(p=0,009)$ e IL $(p=0,019)$ y los votantes del PS tienen valores más altos.

Con relación a la disposición a compartir fake news pro-derecha (SFND), se encuentran diferencias significativas entre votantes de BE con votantes de $\mathrm{CH}(p<0,001)$ y CDS $(p=0,047)$; entre votantes del PCP y partidarios del $\mathrm{CH}(p=0,009)$; entre personas que votaron por CDS y votantes del PAN $(p=$ $0.040)$; entre CH y L $(p=0,003)$, PS $(p<0,001)$, PSD $(p<0,001)$, PAN $(p<0,001)$ e IL $(p=0,005)$. Cabe señalar que para SFND, todos los votantes de derecha tienen valores promedio significativamente más altos y los votantes de $\mathrm{CH}$ tienen una mayor disposición a compartir en comparación con todos los votantes. 
En lo que a la voluntad de compartir noticias pro-izquierda (SNVE) respecta, encontramos diferencias significativas entre los votantes del BE y los del PSD $(p=0,047)$, PAN $(p=0,017)$, IL $(p=0,027)$; entre los votantes del PS con partidarios del PSD $(p=0,013)$, PAN $(p=0,006)$ e IL $(p=0,014)$, con los votantes BE y PS siempre mostrando valores más altos. En cuanto a la voluntad de compartir noticias pro-derecha (SNVD), los votantes BE son significativamente diferentes de los votantes $\mathrm{CH}(p$ $<0,001)$, PSD $(p=0,031)$; los partidarios del PCP, a au vez del $\mathrm{CH}(\mathrm{p}=0,006)$; y los partidarios de $\mathrm{CH}$ se diferencian significativamente de todos los votantes de partidos de izquierda. Además, existen diferencias entre los partidarios del PSD y del PAN $(p=0,028)$. En todos los casos relacionados con el SNVD, los votantes de los partidos de derecha tienen valores significativamente más altos.

\subsection{Asociación entre autoubicación en la escala política de izquierda-derecha, simpatía partidista, consumo y difusión de fake news}

Para estudiar la asociación entre (2) simpatía partidista, (3) autoubicación de los participantes en la escala política izquierda-derecha, creencia y disposición para compartir noticias y fake news, se utilizó el coeficiente de correlación lineal de Pearson (Tabla 5).

Tabla 5. Correlación entre la autoubicación en la escala izquierda-derecha, simpatía por el partido, creencia y disposición para compartir noticias y noticias falsas (r de Pearson)

\begin{tabular}{cccccccc}
\hline & \multicolumn{2}{c}{ Pro-izquierda } & \multicolumn{2}{c}{ Pro-derecha } & \multicolumn{2}{c}{ Simpatía partidista } & $\begin{array}{c}\text { Autoubicación } \\
\text { escala I-D }\end{array}$ \\
& $\begin{array}{c}\text { Fake } \\
\text { News }\end{array}$ & Noticias & $\begin{array}{c}\text { Fake } \\
\text { News }\end{array}$ & Noticias & Derecha & Izquierda & \\
$\begin{array}{c}\text { Auto-ubicación } \\
\text { escala I-D }\end{array}$ & $-0,018$ & $-0,070$ & $0,277^{* *}$ & $0,271^{* *}$ & $0,467^{* *}$ & $-0,473^{* *}$ & - \\
SPD & 0,018 & $-0,006$ & $0,193^{* *}$ & $0,264^{* *}$ & - & $0,139^{* *}$ & $0,467^{* *}$ \\
SPE & 0,058 & $0,153^{* *}$ & $-0,222^{* *}$ & $-0,147^{* *}$ & $0,139^{* *}$ & - & $-0,473^{* *}$ \\
SFNE & $0,558^{* *}$ & $0,377^{* *}$ & $0,362^{* *}$ & $0,262^{* *}$ & 0,067 & $0,180^{* *}$ & $-0,052$ \\
SFND & $0,366^{* *}$ & $0,295^{* *}$ & $0,628^{* *}$ & $0,390^{* *}$ & $0,206^{* *}$ & $-0,052$ & $0,194^{* *}$ \\
SNVE & $0,307^{* *}$ & $0,496^{* *}$ & $0,232^{* *}$ & $0,273^{* *}$ & 0,031 & $0,216^{* *}$ & $-0,098^{* *}$ \\
SNVD & $0,212^{* *}$ & $0,284^{* *}$ & $0,361^{* *}$ & $0,489^{* *}$ & $0,248^{* *}$ & $-0,004$ & $0,172^{* *}$ \\
\hline
\end{tabular}

Fuente: elaboración propia a partir de datos del cuestionario.

Nota: $* p<0,05, * * p<0,01$

Según la Tabla 5, los resultados indican varias correlaciones positivas y negativas significativas. Es importante destacar las asociaciones entre (2) la simpatía del partido y la creencia en noticias y fake news. La simpatía por los partidos de derecha (SPD) se correlaciona con la creencia en fake news a favor de la derecha $(r=0,193 ; p<0,001)$ y noticias a favor de la derecha $(r=0,264 ; p<0,001)$, mientras que la simpatía por los partidos de izquierda (SPE) se asocia positivamente con noticias proizquierda $(r=0,153 ; \mathrm{p}<0,001)$ y negativamente con la creencia en fake news pro-derecha $(r=-0,222$; $p<0,001)$ y noticias a favor de la derecha $(r=-0,147$; $<<0,001)$.

Cabe señalar que no existen correlaciones significativas entre los más simpatizantes de los partidos de izquierda y las fake news pro-izquierda. En cuanto a la propensión a compartir fake news, los resultados indican que existe una correlación positiva entre votantes con mayor simpatía por los partidos de izquierda y el deseo de compartir contenido pro-izquierda; y una correlación positiva entre los partidarios de los partidos de derecha y la voluntad de compartir contenido a favor de la derecha. Sin embargo, no existen asociaciones significativas entre partidarios de partidos de izquierda con el deseo de compartir contenido de derecha y viceversa. 
La (3) autoubicación de los encuestados en la escala política izquierda-derecha también muestra una correlación positiva y significativa con la simpatía partidaria de la derecha $(r=0,467 ; p<0,001)$, con la voluntad de compartir contenido a favor de la derecha (fake news: $r=0,194, p<0,001$; y noticias: $r$ $=0,172 ; p<0,001)$ y creencia en fake news pro-derecha $(r=0,277 ; p<0,001)$ y noticias pro-derecha $(r=0,271 ; p<0,001)$. Por otro lado, la autoubicación en la escala izquierda-derecha tiende a ser contraria a la simpatía partidaria de la izquierda, con una correlación negativa entre las variables $(r=-$ $0,473 ; p<0,001)$. No existe una correlación significativa entre la autoubicación en la escala política y la creencia en fake news y noticias a favor de la izquierda y la voluntad de compartir fake news y noticias pro-izquierda. Si observamos la Tabla 6, vemos la asociación entre la simpatía partidaria, de manera independiente por partido, con la creencia y la voluntad de compartir noticias y fake news.

Tabla 6. Correlación entre la simpatía partidaria (por partido) con la creencia y la voluntad de compartir noticias y fake news (r de Pearson)

\begin{tabular}{|c|c|c|c|c|c|c|c|c|c|}
\hline \multirow{6}{*}{ 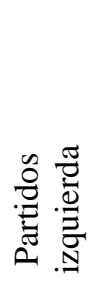 } & & FNE & FND & NVE & NVD & SFNE & SFND & SNVE & SNVD \\
\hline & PCP & $0,083 *$ & $-0,103 * *$ & $0,141^{* *}$ & $-0,085$ & $0,156^{* *}$ & 0,022 & $0,168 * *$ & 0,013 \\
\hline & $\mathrm{BE}$ & 0,044 & $-0,190 * *$ & $0,157^{* *}$ & $-0,105 * *$ & $0,159^{* *}$ & $-0,039$ & $0,215^{* * *}$ & 0,019 \\
\hline & PAN & $-0,012$ & $-0,195 * *$ & 0,019 & $-0,148 * *$ & 0,036 & $-0,093^{*}$ & 0,072 & $-0,065$ \\
\hline & $\mathrm{L}$ & 0,066 & $-0,140 * *$ & $0,106^{* *}$ & $-0,098$ & $0,142 * *$ & $-0,031$ & $0,143^{* *}$ & $-0,014$ \\
\hline & PS & 0,038 & $-0,184 * *$ & $0,148 * *$ & $-0,101 * *$ & $0,176^{* *}$ & $-0,044$ & $0,199 * *$ & 0,032 \\
\hline \multirow{4}{*}{ 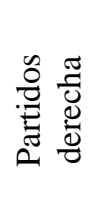 } & PSD & $-0,032$ & $0,078^{*}$ & 0,029 & $0,208 * *$ & 0,059 & $0,141^{* *}$ & 0,036 & $0,220 * *$ \\
\hline & IL & $-0,011$ & $0,087 *$ & $-0,028$ & $0,180 * *$ & 0,034 & $0,112 * *$ & 0,001 & $0,136^{* *}$ \\
\hline & CDS & 0,029 & $0,098^{* *}$ & $-0,017$ & $0,180^{* * *}$ & 0,040 & $0,122^{* *}$ & 0,016 & $0,179 * *$ \\
\hline & $\mathrm{CH}$ & 0,070 & $0,312 * *$ & $-0,007$ & $0,212 * *$ & 0,063 & $0,235^{* *}$ & 0,038 & $0,235^{* *}$ \\
\hline
\end{tabular}

Nota: $* p<0,05, * * p<0,01$

Fuente: elaboración propia a partir de datos del cuestionario.

Según la Tabla 6, solo la simpatía partidista en el PCP tiene una correlación positiva con la creencia en noticias falsas a favor de la izquierda $(r=0,083 ; p=0,026)$. Por otro lado, la creencia en fake news pro-derecha muestra correlaciones positivas con la simpatía en todos los partidos de la derecha, destacando la correlación con $\mathrm{CH}(r=0,312 ; p<0,001)$ y las correlaciones negativas con la simpatía partidaria de la izquierda, revelando un comportamiento contrario. Esta tendencia también se puede ver en la asociación de la simpatía del partido con noticias pro-derecha, aunque no de manera tan explícita. En cuanto a la asociación entre el deseo de compartir, hay un resultado similar, con los partidarios de los partidos de izquierda con asociaciones positivas con SFNE y SNVE, y viceversa.

\section{Discusión y conclusiones}

Nuestro estudio tuvo como objetivo analizar la relación entre el partidismo, como razonamiento motivado, y la creencia y difusión de noticias y fake news políticas. Para evaluar esta relación, fue crucial comparar (1) la intención de voto, (2) la simpatía partidista, y (3) la autoubicación en la escala política de izquierda y derecha con las variables de medición de la creencia y de la voluntad de compartir contenido por los participantes. Nuestros resultados han demostrado que la identidad de partido influye en cómo el electorado consume desinformación. Sin embargo, vemos una mayor tendencia de los participantes partidarios de derecha a aceptar fake news y noticias que confirman sus creencias. Esta tendencia se evidenció con relación a los tres parámetros de medición del partidismo, 
en contraste con lo revelado con los partidarios de izquierda, ya que no hubo diferencias significativas entre votar, autoposicionarse y/o simpatizar con la izquierda y creer en fake news pro-izquierda. Es importante señalar que las fake news a favor de la izquierda no presentaron diferencias significativas para ninguno de los grupos, pero las fake news y las noticias pro-derecha no solo motivan una mayor creencia de los votantes de derecha, sino que parecen estimular una actitud significativamente desigual entre los votantes de izquierda. Los partidarios de izquierda no son indiferentes a las fake news y las noticias a favor de la derecha. En cuanto a la actitud de los encuestados de izquierda hacia el contenido a favor de la derecha (noticias y fake news), comprobamos que los partidarios de izquierda no tienden a creer en las fake news en general, pero son escépticos con las noticias que no son compatibles con sus creencias. Curiosamente, la literatura ha demostrado, sin embargo, que son los conservadores (de derecha) quienes reaccionan de manera más activa a las amenazas o la información negativa (Fessler et al., 2017).

En comparación con los resultados obtenidos por Baptista et al., (2021), podemos confirmar que el partidismo, al igual que la ideología política, pone de manifiesto que las personas que pertenecen a partidos de derecha son más vulnerables a creer en fake news. Sin embargo, Baptista et al., (2021) demostraron que las personas conservadoras y/o que defienden los valores sociales y culturales más a la derecha creían y compartían fake news tanto a favor de la derecha, como a favor de la izquierda. Esto contradice el sesgo de confirmación. Sin embargo, el presente estudio, a diferencia de Baptista et al. (2021), evidenció que los partidarios de derecha son más propensos a aceptar fake news pro-derecha, pero no a aceptar fake news pro-izquierda. Además, refuerza la teoría del sesgo de confirmación por el hecho de que los partidarios de la izquierda tienden a compartir contenido de izquierda y los partidarios de derecha a compartir contenido de derecha. Esto no se demostró en el estudio anterior (Baptista et al., 2021), en el que las personas ideológicamente de derecha también tenían una mayor tendencia a compartir contenido de izquierda. Por lo tanto, con esta investigación verificamos que la identidad de partido ofrece resultados más predecibles y puede ser un mejor predictor que la ideología política. Aun así, estos resultados no coinciden, en parte, con la mayoría de los estudios de la literatura académica consultada, que apuntan al prejuicio partidista como un fuerte predictor de la creencia en fake news compatibles con cada una de las ideologías, tanto de izquierda como de derecha (Allcott y Gentzkow, 2017; Ditto et al., 2019; Faragó et al., 2020; Fessler et al., 2017; Uscinski et al., 2016; Van Bavel y Pereira, 2018). Sin embargo, los partidarios de la izquierda han revelado esta tendencia con respecto a la voluntad de compartir. Además, los partidarios de izquierda y derecha siempre han tenido un comportamiento antagónico.

Aun así, varios estudios reconocen, desde una perspectiva ideológica, que los conservadores son más propensos a creer y difundir fake news (Grinberg et al., 2019; Guess et al., 2019; Roets, 2017). Además, dado que es la orientación anti-izquierdista y anti-gubernamental [3] la que domina la provisión de desinformación en línea en Portugal (Baptista y Gradim, 2020b; Cardoso et al., 2019a; Cardoso et al., 2019b), podemos sugerir que los resultados están en sintonía con lo esperado. Como sucedió en las elecciones estadounidenses, en las que todo indica que se crearon fake news para una audiencia muy específica (conservadora/republicana) (Grinberg et al., 2019; Guess et al., 2019), podemos especular, de este modo, que las personas del espectro político de derecha, también en Portugal, están más expuestas a fake news políticas. Se sabe que la exposición repetida puede hacer que las noticias falsas sean más creíbles (Pennycook y Rand, 2018) ayudar a que la corrección de información falsa sea más difícil de aceptar (Shin y Thorson, 2017), especialmente por personas con altos niveles de autoritarismo (Rollwage et al., 2018) (lo que puede definir a algunas de las personas que simpatizan con $\mathrm{CH}$ ). Gorman y Gorman (2016) sostienen que las personas disfrutan de un "placer genuino" cuando encuentran información que confirma sus creencias, lo que puede ser la base para la creación de burbujas de filtro y cámaras de eco. 
$\mathrm{Al}$ analizar los datos individualmente por partido, destaca el comportamiento de los votantes y simpatizantes del partido de la derecha populista radical (Chega, $\mathrm{CH}$ ) frente a todos los demás. Las personas que se identifican con $\mathrm{CH}$ tienen mayores niveles de creencia y disposición a compartir noticias y fake news que favorecen a la derecha. La evidencia empírica previa ha enfatizado en la creación y difusión de fake news en el ámbito de la derecha radical, especialmente en las democracias occidentales (Bennett y Livingston, 2018; Freelon et al., 2020). Como mencionamos anteriormente, en Portugal, el partido Chega también parece estar asociado con el uso de bots o perfiles falsos en las redes sociales, imponiendo su ideología y desacreditando a fuerzas y figuras políticas de otros partidos mediante el uso de la sátira, la mentira y la distorsión de la verdad, mientras dirige ataques a periodistas y medios tradicionales (Carvalho, 2020b; Silva, 2020). Silva (2020) denuncia la forma en que un "ejército" de perfiles falsos pretende imponer a Chega en una región portuguesa (distrito de Braga), demostrando la forma en que los perfiles falsos están invadiendo grupos de Facebook o comunidades que no están estrechamente relacionadas con ninguna fuerza política o ideológica, pero que reúnen a miles de miembros porque comparten la región o la ciudad donde residen. Además, varias investigaciones periodísticas denunciaron las conexiones de los líderes de Chega con los cultos religiosos, en los que los pastores buscan movilizar a sus fieles para que se unan al partido (Carvalho, 2020a; Público, 2020), como sucedió en Estados Unidos con Donald Trump, con la corriente evangélica ultraconservadora aprobando sus ideales y apoyando sus campañas.

Para terminar, el partidismo provoca que las personas sean más vulnerables a las fake news políticas, moldeando sus actitudes y juicios de acuerdo con sus creencias preexistentes. En Portugal, la identidad de partido tiene una mayor influencia en el electorado de derecha. A la vista de estos resultados, creemos que la desinformación online dirigida principalmente a esta audiencia concreta (antisistema e insatisfecha) probablemente pueda tener un efecto mayor, aunque no se haya evaluado ni estudiado esta influencia.

\section{Notas}

[1] Ver https://bit.ly/36yG4gK (Consejo Europeo)

[2] Ver https://poligrafo.sapo.pt

[3] Desde 2015, el Partido Socialista (PS) dirige el gobierno en Portugal.

\section{Referencias bibliográficas}

Alberti, M. (2020, 28 de Septiembre). Portugal records surge in racist violence as far right rises. The Guardian. https://www.theguardian.com/world/2020/sep/28/portugal-sees-surge-in-racist-violenceas-far-right-rises

Albright, J. (2017). Welcome to the era of fake news. Media and Communication, 5(2), 87-89. https://doi.org/10.17645/mac.v5i2.977

Allcott, H. \& Gentzkow, M. (2017). Social Media and Fake News in the 2016 Election. Journal of Economic Perspectives, 31(2), 211-236. https://doi.org/10.1257/jep.31.2.211

Baptista, J. (2020). Ethos, pathos e logos. Análise comparativa do processo persuasivo das (fake) news. Eikon, 1(7). https://doi.org/10.25768/20.04.04.07.04 
Baptista, João Pedro. \& Gradim, A. (2020a). Understanding Fake News Consumption: A Review. Social Sciences, 9(10), 185. https://doi.org/10.3390/socsci9100185

Baptista, J.P. \& Gradim, A. (2020b). Online disinformation on Facebook: the spread of fake news during the Portuguese 2019 election. Journal of Contemporary European Studies, 1-16. https://doi.org/10.1080/14782804.2020.1843415

Baptista JP, Correia E, Gradim A. \& Piñeiro-Naval V. (2021). The Influence of Political Ideology on Fake News Belief: The Portuguese Case. Publications, 9(2), 23. https://doi.org/10.3390/publications9020023

Belchior, A. M. (2015). Crise económica e perceções sobre a ideologia dos partidos políticos em Portugal (2008-2012). Análise Social, 217, 734-760. https://www.jstor.org/stable/44071983

Benkler, Y., Tilton, C., Etling, B., Roberts, H., Clark, J., Faris, R., Kaiser, J., \& Schmitt, C. (2020). Mail-In Voter Fraud: Anatomy of a Disinformation Campaign. Berkman Center Research Publication No. 2020-6. https://dx.doi.org/10.2139/ssrn.3703701

Bennett, W.L. \& Livingston, S. (2018). The disinformation order: Disruptive communication and the decline of democratic institutions. European Journal of Communication, 33(2), 122-139. https://doi.org/10.1177/0267323118760317

Berger, J. \& Milkman, K. L. (2012). What makes online content viral? Journal of Marketing Research, 49(2), 192-205. https://doi.org/10.1509/jmr.10.0353

Bolsen, T., Druckman, J. N. \& Cook, F. L. (2014). The influence of partisan motivated reasoning on public opinion. Political Behavior, 36(2), 235-262. https://doi.org/10.1007/s11109-013-9238-0

Brauck, M., Diez, G., Kühn, A., Müller, M., Nezik, A. K. \& Steinmetz, V. (2016). Lying press?: Germans lose faith in the fourth estate. Spiegel Online, 24. https://www.spiegel.de/international/germany/most-germans-think-the-press-is-lying-to-themabout-refugees-a-1079049.html (Visitada 16 junio 2021)

Bright, J. (2016). The social news gap: How news reading and news sharing diverge. Journal of Communication, 66(3), 343-365. https://doi.org/10.1111/jcom.12232

Bronstein, M. V, Pennycook, G., Bear, A., Rand, D. G. \& Cannon, T. D. (2019). Belief in fake news is associated with delusionality, dogmatism, religious fundamentalism, and reduced analytic thinking. Journal of Applied Research in Memory and Cognition, 8(1), 108-117. https://doi.org/10.1016/j.jarmac.2018.09.005

Burkhardt, J. M. (2017).History of Fake News. Library Technology Reports, 53(8), 5-9. https://journals.ala.org/index.php/ltr/article/view/6497

Câncio, F. (2020, 27 de Enero). ERC regista como "informativo" site de desinformação e propaganda. Diário de Notícias. https://www.dn.pt/edicao-do-dia/27-jan-2020/erc-regista-como-informativosite-de-desinformacao-e-propaganda-11751353.html

Cardoso, G., Moreno, J. \& Narciso, P. (2019a). Social Media disinformation in the pre-electoral period in Portugal. CIES e-Working Paper N.o 230/2020. https://repositorio.iscte- 
RLCS, Revista Latina de Comunicación Social, 79, 23-47

[Investigación] DOI: 10.4185/RLCS-2021-1509 | ISSN 1138-5820 | Año 2021

iul.pt/bitstream/10071/20667/1/CIES_WP230_Gustavo\%20Cardoso\%2c\%20José\%20Moreno\%2c \%20Inês\%20Narciso\%2c\%20Nuno\%20Palma.pdf

Cardoso, G., Narciso, I., Moreno, J. \& Palma, N. (2019b). Report - Online Disinformation During Portugal's 2019 elections. Lisbon: MEDIALAB \& Democracy Reporting International.

Cardoso, G., Paisana, M. \& Pinto-Martinho, A. (2019c). The Reuters Institute for the Study of Journalism: Reuters Digital News Report 2019 - Portugal. En Publicações OberCom: Portugal.

Cardoso, G., Paisana, M. \& Pinto-Martinho, A. (2020). Reuters Digital News Report 2020 Portugal. En Publicações OberCom: Portugal.

Carreira da Silva. \& Salgado, S.(2018). Why no populism in Portugal? En Lobo, C. M., Carreira da Silva., Zúquete, J. P (Eds.): Citizenship in crisis, Imprensa de Ciências Sociais, 249-268.

Carvalho, M. (2020a, 20 de Mayo). Líderes e pastores evangélicos fazem campanha pelo Chega. Visão. https://visao.sapo.pt/atualidade/politica/2020-05-20-lideres-e-pastores-evangelicos-fazemcampanha-pelo-chega/

Carvalho, M. (2020b, 20 de Mayo). Investigação: Os segredos do pregador Ventura. Visão. https://visao.sapo.pt/atualidade/politica/2020-05-20-investigacao-os-segredos-do-pregador-ventura/

Catarino, J. P. L. (2017). O Pessoas-Animais-Natureza (PAN) como inovação no sistema partidário português [Tesis de Máster]. Instituto Superior de Ciências Sociais e Políticas. Universidade de Lisboa.

Conger, K. (2020, 5 de Noviembre). Tracking Viral Misinformation About the 2020 Election. New York Times.

Costa, J. M. (2011). O Partido Nacional Renovador: a nova extrema-direita na democracia portuguesa. Análise Social, 201, 765-787. https://www.jstor.org/stable/41494872

Da Silva, M. T., Figueiras, R., Brites, M. J., Amaral, I., Maropo, L., Santos, S. C., Jerónimo, P., Santo, P. E. \& Pacheco, L. (2017). Audiências e cross-media: Estudo de padrões de consumo de notícias em Portugal. Estudos Em Comunicacao, 1(25), 177-199. https://doi.org/10.20287/ec.n25.v1.a11

de Almeida, F. C. (2015). A direita radical em Portugal: da Revolução dos Cravos à era da internet. Estudos Ibero-Americanos, 41(1), 98-125. https://doi.org/10.15448/1980-864X.2015.1.20463

Dean, S. (2020, 3 de Noviembre). Twitter flags Trump election tweets as misleading. Los Angeles Times. $\quad$ https://www.latimes.com/business/story/2020-11-03/twitter-trump-2020-election-night$\underline{\text { tweet-disclaimer }}$

Deppe, K. D., Gonzalez, F. J., Neiman, J. L., Jacobs, C., Pahlke, J., Smith, K. B. \& Hibbing, J. R. (2015). Reflective liberals and intuitive conservatives: A look at the Cognitive Reflection Test and ideology. Judgment \& Decision Making, 10(4).

Dilanian, K. (2020, 5 de Noviembre). The Russians have no need to spread misinformation. Trump and his allies are doing it for them. NBC News. https://www.nbcnews.com/politics/2020election/russians-have-no-need-spread-misinformation-trump-his-allies-are-n1246653 
Ditto, P. H., Liu, B. S., Clark, C. J., Wojcik, S. P., Chen, E. E., Grady, R. H., Celniker, J. B. \& Zinger, J. F. (2019). At least bias is bipartisan: A meta-analytic comparison of partisan bias in liberals and conservatives. Perspectives on Psychological Science, 14(2), 273-291. https://doi.org/10.1177/1745691617746796

Faragó, L., Kende, A. \& Krekó, P. (2020). We only Believe in News That We Doctored Ourselves: The Connection between Partisanship and Political Fake News. Social Psychology, 51(2), 77-90. https://doi.org/10.1027/1864-9335/a000391

Fernandes, J. M. \& Magalhaes, P. C. (2020). The 2019 Portuguese general elections. West European Politics, 43(4), 1038-1050. https://doi.org/10.1080/01402382.2019.1702301

Fernández, L. R. (2019). Desinformación y comunicación organizacional: estudio sobre el impacto de las fake news. Revista latina de comunicación social, (74), 1714-1728. https://doi.org/10.4185/RLCS-2019-1406

Fernández García, B. \& Salgado, S. (2020). Populism by the people: An analysis of online comments in Portugal and Spain. International Conference on Social Media and Society, 210-219. https://doi.org/10.1145/3400806.3400831

Fessler, D. M. T., Pisor, A. C. \& Holbrook, C. (2017). Political Orientation Predicts Credulity Regarding Putative Hazards. Psychological Science, 28(5), 651-660. https://doi.org/10.1177/0956797617692108

Fishman, R. M. (2011). Democratic practice after the revolution: the case of Portugal and beyond. Politics \& Society, 39(2), 233-267. https://doi.org/10.1177/0032329211405439

Flaxman, S., Goel, S. \& Rao, J. M. (2016). Filter bubbles, echo chambers, and online news consumption. Public Opinion Quarterly, 80(S1), 298-320. https://doi.org/10.1093/poq/nfw006

Freelon, D., Marwick, A, \& Kreiss, D. (2020). False equivalencies: Online activism from left to right. Science, 369(6508), 1197-1201. https://doi.org/10.1126/science.abb2428

Freire, A. (2017). Para lá da" geringonça": o governo de esquerdas em Portugal e na Europa. Contraponto Editores: Portugal.

Gorman, S. E. \& Gorman, J. M. (2016). Denying to the grave: Why we ignore the facts that will save us. Oxford University Press.

Grinberg, N., Joseph, K., Friedland, L., Swire-Thompson, B. \& Lazer, D. (2019). Fake news on Twitter during the 2016 U.S. presidential election. Science, 363(6425), 374 LP - 378. https://doi.org/10.1126/science.aau2706

Guedes, N. (2012). Convergência ideológica? Uma análise comparada dos programas eleitorais do PS e do PSD (1991-2009). Sociologia, Problemas e Práticas, 68, 103-125. http://journals.openedition.org/spp/711

Guedes, N. (2016). Esquerda-direita: análise das posições ideológicas do PS e do PSD (1990-2010). Sociologia, Problemas e Práticas, 80, 95-116. http://journals.openedition.org/spp/2110 
Guess, A., Nagler, J. \& Tucker, J. (2019). Less than you think: Prevalence and predictors of fake news dissemination on Facebook. Science Advances, 5(1), eaau4586. https://doi.org/10.1126/sciadv.aau4586

Hallin, D. C. \& Mancini, P. (2017). Ten Years After Comparing Media Systems: What Have We Learned? Political Communication, $\quad 34(2), \quad 155-171$. https://doi.org/10.1080/10584609.2016.1233158

Halpern, D., Valenzuela, S., Katz, J. \& Miranda, J. P. (2019). From Belief in Conspiracy Theories to Trust in Others: Which Factors Influence Exposure, Believing and Sharing Fake News. International Conference on Human-Computer Interaction, 217-232. https://doi.org/10.1007/978-3$\underline{030-21902-4 \_16}$

Humprecht, E. (2019). Where 'fake news' flourishes: a comparison across four Western democracies. Information Communication and Society, 22(13), 1973-1988. https://doi.org/10.1080/1369118X.2018.1474241

ISCTE. (2019). Disinformation risks in Portugal's election more Brazil than Europe? Risk assessment: online manipulation ahead of the portuguese parliamentary elections. https://medialab.iscteiul.pt/desinformacao-risco-de-interferencia-nas-eleicoes-e-reduzido-2

Jalali, C. \& Cabral, R. (2003). A investigação do comportamento eleitoral em Portugal: história e perspectivas futuras. Análise Social, 545-572. https://www.jstor.org/stable/41012401

Jalali, C., Moniz, J. \& Silva, P. (2021). In the Shadow of the 'Government of the Left': The 2019 Legislative Elections in Portugal. South European Society and Politics, 1-27. https://doi.org/10.1080/13608746.2020.1868702

Jost, J. T., Glaser, J., Kruglanski, A. W. \& Sulloway, F. J. (2003). Political conservatism as motivated social cognition. Psychological Bulletin, 129(3), 339. https://doi.org/10.1037/0033-2909.129.3.339

Lazer, D. M. J., Baum, M. A., Benkler, Y., Berinsky, A. J., Greenhill, K. M., Menczer, F., Metzger, M. J., Nyhan, B., Pennycook, G., Rothschild, D., Schudson, M., Sloman, S. A., Sunstein, C. R., Thorson, E. A., Watts, D. J. \& Zittrain, J. L. (2018). The science of fake news. Science, 359(6380), 1094 LP. https://doi.org/10.1126/science.aao2998

Lee, E., Karimi, F., Wagner, C., Jo, H.-H., Strohmaier, M. \& Galesic, M. (2019). Homophily and minority-group size explain perception biases in social networks. Nature Human Behaviour, 3(10), 1078-1087. https://doi.org/10.1038/s41562-019-0677-4

Leeper, T. J. \& Slothuus, R. (2014). Political parties, motivated reasoning, and public opinion formation. Political Psychology, 35, 129-156. https://doi.org/10.1111/pops.12164

Lewandowsky, S., Ecker, U. K. H. \& Cook, J. (2017). Beyond misinformation: Understanding and coping with the "post-truth" era. Journal of Applied Research in Memory and Cognition, 6(4), 353369. https://doi.org/10.1016/j.jarmac.2017.07.008

Lewis, R. \& Marwick, A. E. (2017). Taking the red pill: Ideological motivations for spreading online disinformation. Understanding and Addressing the Disinformation Ecosystem, 18-22. 
RLCS, Revista Latina de Comunicación Social, 79, 23-47

[Investigación] DOI: 10.4185/RLCS-2021-1509 | ISSN 1138-5820 | Año 2021

https://firstdraftnews.org/wp-content/uploads/2018/03/The-Disinformation-Ecosystem-20180207v2.pdf

Lisi, M. (2013). Rediscovering civil society? Renewal and continuity in the Portuguese radical left. South European Society and Politics, 18(1), 21-39. https://doi.org/10.1080/13608746.2012.757450

Lisi, M. (2016). U-turn: the Portuguese radical left from marginality to government support. South European Society and Politics, 21(4), 541-560. https://doi.org/10.1080/13608746.2016.1225331

Lisi, M. (2019). Portugal: Defeat for the right, challenges for the left. En Sio, L., Franklin, M., Russo, L. (Eds.) The European Parliament Elections of 2019, Luiss University Press: Rome, Italy, 225230.

Lisi, M., Sanches, E. R. \& dos Santos Maia, J. (2021). Party System Renewal or Business as Usual? Continuity and Change in Post-Bailout Portugal. South European Society and Politics, 1-25. https://doi.org/10.1080/13608746.2020.1862498

Lorenz-Spreen, P., Lewandowsky, S., Sunstein, C. R. \& Hertwig, R. (2020). How behavioural sciences can promote truth, autonomy and democratic discourse online. Nature Human Behaviour, 4(11), 1102-1109. https://doi.org/10.1038/s41562-020-0889-7

March, L. (2012). Os partidos europeus da esquerda radical após a crise: Marxismo, mainstream ou marginalidade. En L. March \& A. Freire (Eds.), A Esquerda Radical em Portugal e na Europa: Marxismo, Mainstream ou Marginalidade (QuidNovi, 25-104).

Marchi, R. (2019, 21 de Diciembre). Um olhar exploratório sobre o partido Chega. Observador. https://observador.pt/opiniao/um-olhar-exploratorio-sobre-o-partido-chega/

Marwick, A. E. (2018). Why do people share fake news? A sociotechnical model of media effects. Georgetown Law Technology Review, 2(2), 474-512.

Mendes, M. S. \& Dennison, J. (2020). Explaining the emergence of the radical right in Spain and Portugal: salience, stigma and supply. West European Politics, 1-24. https://doi.org/10.1080/01402382.2020.1777504

MMF. (2021). Portugal: SIC journalists receive torrent of abuse and threats online after Chega investigation. https://mappingmediafreedom.ushahidi.io/posts/23757

Nielsen, R. K. \& Graves, L. (2017). “News you don't believe”: Audience perspectives on fake news. https://reutersinstitute.politics.ox.ac.uk/sites/default/files/201710/Nielsen\&Graves_factsheet_1710v 3_FINAL_download.pdf

Nyhan, B. \& Reifler, J. (2010). When corrections fail: The persistence of political misperceptions. Political Behavior, 32(2), 303-330. https://doi.org/10.1007/s11109-010-9112-2

Ordaz, L. V., Calvo, D. \& García, G. L. (2018). Conversaciones políticas en Facebook. Explorando el papel de la homofilia en la argumentación y la interacción comunicativa. Revista Latina de Comunicación Social, (73), 55-73. https://doi.org/ 10.4185/RLCS-2018-1245

Pariser, E. (2011). The filter bubble: What the Internet is hiding from you. Penguin UK. 
Pena, P. (2018a, 11 de Noviembre). Fake News: sites portugueses com mais de dois milhões de seguidores. Diário de Notícias. https://www.dn.pt/edicao-do-dia/11-nov-2018/fake-news-sitesportugueses-com-mais-de-dois-milhoes-de-seguidores--10160885.html

Pena, P. (2018b, 25 de Noviembre). O negócio da desinformação: empresa canadiana faz fake news em Portugal. Diário de Notícias. https://www.dn.pt/edicao-do-dia/25-nov-2018/o-negocio-dadesinformacao-empresa-canadiana-faz-fake-news-em-portugal--10231174.html

Pena, P. (2019a, Abril 7). Chega e Iniciativa Liberal dominam campanha nas redes sociais. Diário de Notícias. $\quad$ https://www.dn.pt/edicao-do-dia/07-abr-2019/chega-e-iniciativa-liberal-dominamcampanha-nas-redes-sociais-10768894.html

Pena, P. (2019b). Fábrica de mentiras. Viagem ao mundo das Fake News. Objectiva, Portugal.

Pennycook, G. \& Rand, D. G. (2019). Lazy, not biased: Susceptibility to partisan fake news is better explained by lack of reasoning than by motivated reasoning. Cognition, 188, 39-50. https://doi.org/10.1016/j.cognition.2018.06.011

Pennycook, G. \& Rand, D. G. (2019). Who falls for fake news? The roles of bullshit receptivity, overclaiming, familiarity, and analytic thinking. Journal of Personality. https://doi.org/10.1111/jopy.12476

Pennycook, G. \& Rand, D. G. (2021). Research note: Examining false beliefs about voter fraud in the wake of the 2020 Presidential Election. Harvard Kennedy School Misinformation Review. https://misinforeview.hks.harvard.edu/article/research-note-examining-false-beliefs-about-voterfraud-in-the-wake-of-the-2020-presidential-election/

Pereira, A. \& Van Bavel, J. (2018). Identity concerns drive belief in fake news. PsyArXiv Working Paper. http://dx.doi.org/10.31234/OSF.IO/7VC5D

Pereira, J. S. (2016). A esquerda radical no período pós-2009: nada de (muito) novo em Portugal? Oficina Do Historiador, 9(1), 58-77. https://doi.org/10.15448/2178-3748.2016.1.22961

Público. (2020, 21 de Mayo). Pastores evangélicos promovem André Ventura. Público. https://www.publico.pt/2020/05/21/politica/noticia/pastores-evangelicos-promovem-andre-ventura$\underline{1917509}$

Recuero, R. \& Gruzd, A. (2019). Cascatas de Fake News Políticas: um estudo de caso no Twitter. Galáxia (São Paulo), 41, 31-47. https://doi.org/10.1590/1982-25542019239035

Roets, A. (2017). 'Fake news': Incorrect, but hard to correct. The role of cognitive ability on the impact of false information on social impressions. Intelligence, 65, 107-110. https://doi.org/10.1016/j.intell.2017.10.005

Rollwage, M., Dolan, R. J. \& Fleming, S. M. (2018). Metacognitive failure as a feature of those holding radical beliefs. Current Biology, 28(24), 4014-4021. https://doi.org/10.1016/j.cub.2018.10.053 
Russell, A. (2019). 'This time it's different': Covering threats to journalism and the eroding public sphere. Journalism, 20(1), 32-35. https://doi.org/10.1177/1464884918809245

Santana-Pereira, J. \& Cancela, J. (2021). Demand without Supply? Populist Attitudes and Voting Behaviour in Post-Bailout Portugal. South European Society and Politics, 1-24. https://doi.org/10.1080/13608746.2020.1864910

Sartori, G. (2005). Parties and party systems: A framework for analysis. ECPR press.

Shin, J. \& Thorson, K. (2017). Partisan selective sharing: The biased diffusion of fact-checking messages on social media. Journal of Communication, 67(2), 233-255. https://doi.org/10.1111/jcom.12284

Silva, P. L. (2020, 27 de Mayo). Como um “exército" de perfis falsos quer impor o Chega em Braga. $O$ Minho. https://ominho.pt/como-um-exercito-de-perfis-falsos-quer-impor-o-chega-em-braga/

Silverman, C. \& Singer-Vine, J. (2016, 6 de Deciembre). Most Americans who see fake news believe

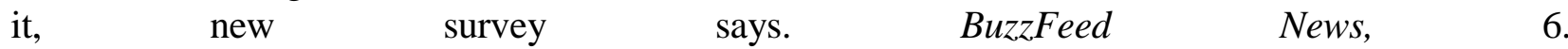
https://www.buzzfeednews.com/article/craigsilverman/fake-news-survey

Silverman, C., Strapagiel, L., Shaban, H., Hall, E. \& Singer-Vine, J. (2016, 20 de Octubre). Hyperpartisan Facebook pages are publishing false and misleading information at an alarming rate. Buzzfeed News, 20, 68. https://www.buzzfeednews.com/article/craigsilverman/partisan-fb-pagesanalysis

Swift, A. (2016, 14 de Septiembre). Americans' trust in mass media sinks to new low. Gallup News, 14. https://news.gallup.com/poll/195542/americans-trust-mass-media-sinks-new-low.aspx

Swire, B., Berinsky, A. J., Lewandowsky, S., \& Ecker, U. K. H. (2017). Processing political misinformation: comprehending the Trump phenomenon. Royal Society Open Science, 4(3). https://doi.org/10.1098/rsos.160802

Uscinski, J. E., Klofstad, C. \& Atkinson, M. D. (2016). What drives conspiratorial beliefs? The role of informational cues and predispositions. Political Research Quarterly, 69(1), 57-71. https://doi.org/10.1177/1065912915621621

Van Bavel, J. J. \& Pereira, A. (2018). The Partisan Brain: An Identity-Based Model of Political Belief. Trends in Cognitive Sciences, 22(3), 213-224. https://doi.org/10.1016/j.tics.2018.01.004

Waisbord, S. (2018). Truth is What Happens to News: On journalism, fake news, and post-truth. Journalism Studies, 19(13), 1866-1878. https://doi.org/10.1080/1461670X.2018.1492881

Wendling, M. (2018, 22 de Enero). The (almost) complete history of "fake news." BBC Trending. https://www.bbc.com/news/blogs-trending-42724320 


\section{AUTORES}

\section{João Pedro Baptista}

João Pedro Baptista es periodista e investigador de la Unidad de Investigación Labcom Comunicación y Artes, del Departamento de Comunicación, Filosofía y Política de la Universidad de Beira Interior, en Portugal. Tiene una maestría en Ciencias de la Comunicación: Comunicación Pública, Política e Intercultural de la Universidad de Trás-os-Montes y Alto Douro y actualmente disfruta de una beca nacional de doctorado en Ciencias de la Comunicación, de la Fundación para la Ciencia y la Tecnología (FCT) de Portugal, en la Universidad de Beira Interior. Los intereses de su investigación están relacionados con el creciente problema de las fake news y la desinformación online, así como el estudio de la situación de la dicotomía política Izquierda-Derecha enmarcada con el estudio de la comunicación política.

joao.pedro.baptista@ubi.pt

Orcid ID: https://orcid.org/0000-0002-3684-1923

Google Scholar: https://scholar.google.com/citations?user=BBvTAgsAAAAJ\&hl=pt-PT

\section{Elisete Correia}

Elisete Correia es licenciada en estadística, tiene una maestría y un doctorado en probabilidad y estadística de la Facultad de Ciencias de la Universidad de Lisboa. Actualmente, es professora adjunto en la Universidad de Trás-os-Montes y Alto Douro y miembro del Centro de Matemática Computacional y Estocástica (CEMAT), Unidad de Investigación del Instituto Superior Técnico de la Universidad de Lisboa y colaboradora del Centro de Investigación Química - Vila Real. Opera en el área de ciencias exactas con énfasis en Estadística. Publicó varios artículos en revistas científicas internacionales, en artículos técnicos y en actas de congresos. También es revisor de algunas revistas internacionales. Sus líneas de investigación son estadística multivariante, modelado de ecuaciones estructurales, análisis sensorial, diseño experimental.

ecorreia@utad.pt

Orcid ID: https://orcid.org/0000-0002-1121-2792

Google Scholar: https://scholar.google.com/citations?hl=pt-PT\&user=V1_S0C8AAAAJ\&view_op

\section{Anabela Gradim}

Anabela Gradim es licenciada en Filosofía por la Universidad de Porto. Tiene una maestría, un doctorado y una agregación en Ciencias de la Comunicación de la Universidad de Beira Interior (UBI). Docente de Periodismo, Comunicación y Metodología en la Facultad de Artes y Letras de la UBI. Es coordinadora de la unidad de investigación Labcom - Comunicación, Filosofía y Política, y directora del Doctorado en Ciencias de la Comunicación de la UBI. Sus intereses de investigación son el periodismo, la comunicación científica, la semiótica, la retórica y la interfaz de estas disciplinas con la cibercultura y los medios digitales. Coordinó y participó como investigadora en doce proyectos de investigación, y es autora de libros, capítulos de libros y artículos en las áreas de periodismo, semiótica y comunicación científica.

agradim@ubi.pt

Orcid ID: https://orcid.org/0000-0001-6968-1957

Google Scholar: https://scholar.google.com/citations?user=UZL4cFAAAAAJ\&hl=pt-PT\&oi=ao

\section{Valeriano Piñeiro-Naval CV}

Valeriano Piñeiro-Naval es doctor (con mención europea) en Comunicación Audiovisual, Revolución Tecnológica y Cambio Cultural por la Universidad de Salamanca (2015). En la actualidad ejerce como profesor ayudante doctor en el Departamento de Sociología y Comunicación de esta misma institución. Ha trabajado como investigador post-doctoral en la Universidad Rey Juan 
Carlos (Madrid) y en la Universidade da Beira Interior (Covilhã, Portugal), donde disfrutó de una beca nacional de la Fundação para a Ciência e a Tecnologia (FCT). Por otro lado, realizó estancias en la Pontificia Universidad Católica de Chile (Santiago, Chile) y en la Universidad Autónoma de Nuevo León (Monterrey, México). Asimismo, es miembro del Observatorio de los Contenidos Audiovisuales (OCA)

vale.naval@usal.es

Orcid ID: https://orcid.org/0000-0001-9521-3364

Google Scholar: https://scholar.google.com/citations?user=Yp9j2u8AAAAJ\&hl=pt-PT 